\title{
Pro: continuous positive airway pressure and cardiovascular prevention
}

\author{
Miguel Angel Martinez-Garcia ${ }^{1}$, Francisco Campos-Rodriguez², \\ Shahrokh Javaheri ${ }^{3}$ and David Gozal ${ }^{4}$
}

Affiliations: ${ }^{1}$ Pulmonary Dept, Polytechnic and University La Fe Hospital, Valencia, Spain. ${ }^{2}$ Respiratory Dept, Valme University Hospital, Seville, Spain. ${ }^{3}$ Pulmonary and Sleep Division, Bethesda North Hospital, Cincinnati, $\mathrm{OH}$, USA. ${ }^{4}$ Dept of Pediatrics, University of Chicago, Chicago, IL, USA.

Correspondence: Miguel Angel Martinez-Garcia, Pneumology Dept, Hospital Universitario y Politécnico La Fe, Bulevar Sur s/n, 46012-Valencia, Spain. E-mail: mianmartinezgarciadggmail.com

@ERSpublications

The methodological limitations of the current RCTs on the CPAP effect on cardiovascular protection do not support a change in our clinical practice http://ow.ly/V11730ieGYk

Cite this article as: Martinez-Garcia MA, Campos-Rodriguez F, Javaheri S, et al. Pro: continuous positive airway pressure and cardiovascular prevention. Eur Respir J 2018; 51: 1702400 [https://doi.org/10.1183/ 13993003.02400-2017].

In the clinical realm, the highest degree of evidence driving practice guidelines originates from randomised controlled trials (RCTs) and meta-analysis of RCTs. For practice changes to occur, adequate RCT methodology and optimal sample size, as well as accurate definition of primary objectives and patient selection, are paramount, along with satisfactory adherence to the targeted intervention in a high proportion of the participants. Critically, the results of any of such trials can only apply to the targeted population and cannot be extrapolated to alternative groups, even if they suffer from the same disease [1]. Furthermore, if controversial findings emerge between previous basic and clinical studies, a cogent explanation for such discrepancies would be required to identify future study directions.

The majority of published studies on obstructive sleep apnoea (OSA) suggest that it is an independent cardiovascular risk factor [2]. Obstructive events during sleep lead to intermittent hypoxia, large swings in negative intrathoracic pressures, sleep fragmentation with arousals and increased sympathetic tone $[2,3]$. Large population-based and clinical referral cohort studies show that untreated severe OSA is associated with a high risk of incident hypertension, stroke and cardiovascular mortality [4-11]. Conversely, continuous positive airway pressure (CPAP) therapy counteracts most of these intermediate pathophysiological pathways $[2,12-15]$ and would be anticipated to improve cardiovascular outcomes in adherent patients [2]. However, except for hypertension [16, 17], the potential beneficial effects of CPAP therapy on other defined cardiovascular outcomes remain undefined, principally because most trials used an observational design, an approach that precludes the highest level of evidence but is dictated by ethical considerations of withholding treatment in symptomatic OSA patients in an RCT.

Recently, three RCTs [18-20] and a meta-analysis [21] attempted to shed light on the impact of OSA treatment on cardiovascular outcomes. The meta-analysis [21] concluded that positive airway pressure (PAP) therapy provides noncardiovascular benefits, namely improved quality of life and daytime sleepiness, but fails to prevent cardiovascular outcomes. The three aforementioned RCTs included in this meta-analysis yielded similar conclusions, i.e. intention-to-treat analysis does not support the contention that CPAP reduces cardiovascular risk [18-20].

These findings prompted the question, should we change our current clinical practice and not prescribe CPAP therapy in asymptomatic OSA patients, even if these patients are at high cardiovascular risk? If the 
RCT evidence [18-20] was undisputable, there is little if any doubt that such recommendations would be rapidly incorporated into current clinical practice guidelines. However, in our opinion, this is not the case.

First, the three RCTs have methodological particularities that preclude extrapolation of the results to every patient with OSA. Importantly, their primary endpoint consisted of a composite of cardiovascular outcomes including disparate items such as cardiovascular death and a list of nonfatal cardiovascular and cerebrovascular events. Thus, these RCTs were operating under the (false) assumption that all of the singular cardiovascular outcomes were equivalent and interchangeable in terms of their degree of association with OSA or response to CPAP therapy. For instance, patients with coronary heart disease may develop protective collateral vessels in response to OSA-related hypoxia, so this subgroup may have a lower cardiovascular risk [22] and CPAP would have limited therapeutic effect in this population. Therefore, including coronary heart disease in a composite outcome may dampen the potential impact of CPAP. Meanwhile, the SAVE trial [18], which provided the largest sample size, and thus had the greatest influence (73\% of events) on the meta-analysis, had limitations [2]. Paucisymptomatic patients, mostly Asian (62\%), were enrolled. The dropout rate (from the initial assessment for eligibility to the end of the study) was $83 \%$ and 57 patients allocated to the control group switched to CPAP treatment, probably diluting CPAP effect in the intention to treat analysis. In this trial, sleep disordered breathing was assessed using a device acceptable for screening but not for diagnostic purposes, particularly in subjects with cardiovascular disease, according to the latest American Academy of Sleep Medicine guidelines [23]. Moreover, even though patients with pattern of Cheyne-Stokes breathing were excluded, this device was incapable of distinguishing OSA from central sleep apnoea (CSA). Potentially, additional patients with CSA were missed, which should have been an exclusion criterion, and CSA may not be suppressed by CPAP, resulting in excess mortality [2]. In addition, the inclusion of patients with nonsevere OSA in the trial may have further diluted CPAP effects, which are most pronounced in severe OSA [2, 24]. Finally, the aforementioned meta-analysis [21] analysed heterogeneous trials pooling data from studies assessing both OSA and CSA, as well as studies that used different types of PAP treatment, not only CPAP. Thus, bearing in mind the particularities of the RCTs and the limitations of the meta-analysis, one wonders whether the majority of the patients usually seen in daily clinical practice in sleep centres would fulfil the skewed clinical features of the subjects included in these studies and, therefore, legitimise the putative recommendations of the meta-analysis; we believe not.

Second, although CPAP is, overall, a safe therapy without major side-effects, and would be unlikely to benefit and be effective for all OSA patients, strong arguments are required to explain the absolute lack of benefit from CPAP treatment as inferred by the meta-analysis and RCTs. This is all the more disconcerting considering that CPAP therapy abolishes obstructive respiratory events, annulling the pathophysiological consequences of OSA [2, 12-15]. The normalisation of these breathing events with CPAP leads to significant improvement in hypertension $[2,16,17]$ and therefore, commensurate with the benefits of blood pressure reductions on incident cardiovascular outcomes, detectable and beneficial effects on at least some cardiovascular outcomes related to OSA would be anticipated [25].

Third, a therapy can only be effective if it is adequately employed. However, this was not the case. The CPAP group in most of the RCTs failed to adequately adhere to the treatment, with compliance rates lower than $4 \mathrm{~h}$ per night. Although the minimal threshold of adherence associated with cardiovascular protection is unknown, greater compliance rates in minimally symptomatic patients could be necessary to achieve meaningful blood pressure reduction [19, 26, 27]. Importantly, better adherence rates are associated with greater decreases in blood pressure [28]. Thus, similar levels of adherence would be needed to reduce the risk of hard cardiovascular outcomes in minimally symptomatic OSA, particularly with less severe OSA, enrolled in these trials.

Rapid eye movement (REM) sleep OSA has been associated with increased risk of hypertension [29] and cardiovascular disease [30], so selective nonuse of CPAP during these critical periods (i.e. patients who take off the mask after the first hours, remaining untreated during the latter hours of nocturnal sleep when REM dominates and OSA worsens) may account for reduced treatment efficacy. Indeed, to overcome the ethical issues of withholding treatment in sleepy OSA patients, most RCTs recruited minimally symptomatic individuals, an approach that not only excluded the most vulnerable sleepy patients prone to OSA-related cardiovascular outcomes [2] but could also have also influenced the overall poor adherence rates. In fact, when the analyses were restricted to CPAP use $\geqslant 4 \mathrm{~h}$ per night, significant decreases in the composite cardiovascular risk or cerebrovascular risk endpoints emerged [2, 31], thereby raising the concern of whether the lack of cardiovascular benefits in the intention-to-treat analysis is attributable to a real lack of effect of CPAP or can be simply ascribed to poor adherence to therapy. Therefore, the question of whether CPAP will reduce cardiovascular risk in patients with adequate treatment adherence has not yet been answered and this issue is of paramount importance for the formulation of recommendations for sound clinical practice. 
An additional conundrum emerges from the putative conclusions of $\mathrm{YU}$ et al. [21]. Although it is clear that the aforementioned evidence confirms that CPAP therapy is not effective in reducing cardiovascular risk in all OSA patients, it would also be risky to disregard any potential beneficial cardiovascular effect by recommending not to treat OSA patients simply on the basis of studies that do not represent the typical patients referred to sleep clinics and who do not adequately adhere to the treatment.

Before changing our clinical practice and adopting any of the aforementioned recommendations, we strongly advocate carrying out pragmatic trials enrolling patients who are routinely evaluated in real-world clinical practice [32], with a well-balanced geographical representation. These trials should be assessed by a multidisciplinary worldwide expert panel, use consensus-based inclusion/exclusion criteria that are based on medical, biological and ethical equipoise, and employ standardised technologies for detection of both sleep-related and cardiovascular events. Initial focus on well-phenotyped patients with severe OSA and stringently defined individual cardiovascular endpoints at appropriate timeframes, in either primary or secondary prevention, while avoiding composite outcomes [33] (e.g. ischaemic heart disease, stroke and atrial fibrillation) are further suggested, along with adequate statistical power to encompass both intention-to-treat and per protocol analyses as well as sensitivity analyses of clinically relevant subgroups such as sex, age, sleepiness and control/uncontrolled cardiovascular risk factors, especially arterial hypertension and diabetes. The ethical concerns around randomisation of sleepy patients to an untreated arm with lengthy trial follow-up preclude the adoption of ideal trial methodologies, and may require alternative therapeutic approaches and compare their effectiveness with CPAP [34]. Randomising patients from cardiovascular clinics never referred to sleep units to either a sleep study or none presents a potential alternative [35]. Sequential randomisation might also be used to reduce costs and unnecessary exposure to treated/untreated patients [36]. Biological sample collection from all patients is critical to enable in-depth exploration of underpinning pathophysiological mechanisms and formulation of personalised medicine approaches. Finally, in order to improve adherence, we should combine different strategies including educational, supportive and behavioural interventions, use of telemonitoring and telemedicine, and even implementation of financial incentives. An additional approach used in the PARADIGM trial [37], the largest RCT in heart failure, is the insertion of long run-in periods with CPAP before randomisation in order to identify and exclude from randomisation those patients with persistent side-effects and those who are intolerant to CPAP, thereby potentially improving long-term adherence in the study. Considering the elevated prevalence of OSA in the general population [38], feasibility of such studies is likely in the setting of multinational collaborative research groups [39].

Meanwhile, we advocate a more prudent approach, i.e. individualisation of each patient such as to exclude from CPAP only those patients in whom the benefit of CPAP is deemed marginal or nonexistent, and weighing the risk/benefit ratio of such an approach. Considering that, at present, these patients are difficult to identify and in light of the fundamental premise of primum non nocere in our profession, the choice is clear and favours CPAP until proven otherwise.

Conflict of interest: None declared.

\section{References}

1 Umscheid CA, Margolis DJ, Grossman CE. Key concepts of clinical trials: a narrative review. Postgrad Med 2011; 123: 194-204.

2 Javaheri S, Barbe F, Campos-Rodriguez F, et al. Sleep apnea. Types, mechanisms, and clinical cardiovascular consequences. J Am Coll Cardiol 2017; 69: 841-858.

3 Dempsey JA, Veasey SC, Morgan BJ, et al. Pathophysiology of sleep apnea. Physiol Rev 2010; 90: 47-112.

4 Marin JM, Agusti A, Villar I, et al. Association between treated and untreated obstructive sleep apnea and risk of hypertension. JAMA 2012; 307: 2169-2176.

5 Redline S, Yenokyan G, Gottlieb DJ, et al. Obstructive sleep apnea-hypopnea and incident stroke: the Sleep Heart Health Study. Am J Respir Crit Care Med 2010; 182: 269-277.

6 Marshall NS, Wong KK, Liu PY, et al. Sleep apnea as an independent risk factor for all-cause mortality: the Busselton Health Study. Sleep 2008; 31: 1079-1085.

7 Punjabi NM, Caffo BS, Goodwin JL, et al. Sleep-disordered breathing and mortality: a prospective cohort study. PLoS Med 2009; 6: e1000132.

8 Young T, Finn L, Peppard PE, et al. Sleep disordered breathing and mortality: eighteen-year follow-up of the Wisconsin sleep cohort. Sleep 2008; 31: 1071-1078.

9 Marin JM, Carrizo SJ, Vicente E, et al. Long-term cardiovascular outcomes in men with obstructive sleep apnoea hypopnoea with or without treatment with continuous positive airway pressure: an observational study. Lancet 2005; 365: 1046-1053.

10 Campos-Rodriguez F, Martinez-Garcia MA, de la Cruz-Moron I, et al. Cardiovascular mortality in women with obstructive sleep apnea with or without continuous positive airway pressure treatment: a cohort study. Ann Intern Med 2012; 156: 115-122.

11 Martínez-García MA, Campos-Rodríguez F, Catalán-Serra P, et al. Cardiovascular mortality in obstructive sleep apnea in the elderly: role of long-term continuous positive airway pressure treatment: a prospective observational study. Am J Respir Crit Care Med 2012; 186: 909-916. 
12 Schwarz EI, Puhan MA, Schlatzer C, et al. Effect of CPAP therapy on endothelial function in obstructive sleep apnoea: a systematic review and meta-analysis. Respirology 2015; 20: 889-895.

13 Iftikhar IH, Hoyos CM, Phillips CL, et al. Meta-analyses of the association of sleep apnea with insulin resistance, and the effects of CPAP on HOMA-IR, adiponectin, and visceral adipose fat. J Clin Sleep Med 2015; 11: 475-485.

14 Ziegler MG, Mills PJ, Loredo JS, et al. Effect of continuous positive airway pressure and placebo treatment on sympathetic nervous activity in patients with obstructive sleep apnea. Chest 2001; 120: 887-893.

15 Sanchez de la Torre M, Campos-Rodriguez F, Barbe F. Obstructive sleep apnoea and cardiovascular disease. Lancet Respir Med 2013; 1: 61-72.

16 Iftikhar IH, Valentine CW, Bittencourt LR, et al. Effects of continuous positive airway pressure on blood pressure in patients with resistant hypertension and obstructive sleep apnea: a meta-analysis. J Hypertens 2014; 32: 2341-2350.

17 Montesi SB, Edwards BA, Malhotra A, et al. The effect of continuous positive airway pressure treatment on blood pressure: a systematic review and meta-analysis of randomized controlled trials. J Clin Sleep Med 2012; 8: 587-596.

18 McEvoy RD, Antic NA, Heeley E, et al. CPAP for prevention of cardiovascular events in obstructive sleep apnea. N Engl J Med 2016; 375: 919-931.

19 Barbé F, Durán-Cantolla J, Sánchez de-la-Torre M, et al. Effect of continuous positive airway pressure on the incidence of hypertension and cardiovascular events in nonsleepy patients with obstructive sleep apnea: a randomized controlled trial. JAMA 2012; 307: 2161-2168.

20 Peker Y, Glantz H, Eulenburg C, et al. Effect of positive airway pressure on cardiovascular outcomes in coronary artery disease patients with non-sleepy obstructive sleep apnea: the RICCADSA randomized controlled trial. Am J Respir Crit Care Med 2016; 194: 613-620.

$21 \mathrm{Yu}$ J, Zhou Z, McEvoy RD, et al. Association of positive airway pressure with cardiovascular events and death in adults with sleep apnea. A systematic review and meta-analysis. JAMA 2017; 318: 156-166.

22 Steiner S, Schueller PO, Schulze V, et al. Occurrence of coronary collateral vessels in patients with sleep apnea and total coronary occlusion. Chest 2010; 137: 516-520.

23 Kapur VK. Clinical practice guideline for diagnostic testing for adult obstructive sleep apnea: an American Academy of Sleep Medicine clinical practice guideline. J Clin Sleep Med 2017; 13: 479-504.

24 Ge X, Han F, Huang Y, et al. Is obstructive sleep apnea associated with cardiovascular and all-cause mortality? PLoS One 2013; 8: e69432.

25 Mancia G, Fagard R, Narkiewicz K, et al. 2013 Practice guidelines for the management of arterial hypertension of the European Society of Hypertension (ESH) and the European Society of Cardiology (ESC) ESH/ESCTask Force for the Management of Arterial Hypertension. J Hypertens 2013; 31: 1925-1938.

26 Martínez-García MA, Capote F, Campos-Rodríguez F, et al. Effect of CPAP on blood pressure in patients with obstructive sleep apnea and resistant hypertension: The HIPARCO randomized clinical trial. JAMA 2013; 310: 2407-2415.

27 Bratton DJ, Stradling JR, Barbé F, et al. Effect of CPAP on blood pressure in patients with minimally symptomatic obstructive sleep apnoea: a meta-analysis using individual patient data from four randomized controlled trials. Thorax 2014; 69: 1128-1135.

28 Bratton DJ, Gaisl T, Wons AM, et al. CPAP vs mandibular advancement devices and blood pressure in patients with obstructive sleep apnea: a systematic review and meta-analysis. JAMA 2015; 314: 2280-2293.

29 Mokhlesi B, Finn LA, Hagen EW, et al. Obstructive sleep apnea during REM sleep and hypertension. Results of the Wisconsin Sleep Cohort. Am J Respir Crit Care Med 2014; 190: 1158-1167.

30 Mokhlesi B, Varga AW. Obstructive sleep apnea and cardiovascular disease. REM sleep matters! Am J Respir Crit Care Med 2018; 197: 554-556.

31 Abuzaid AS, Al Ashry HS, Elbadawi A, et al. Meta-analysis of cardiovascular outcomes with continuous positive airway pressure therapy in patients with obstructive sleep apnea. Am J Cardiol 2017; 120: 693-699.

32 Saturni S, Bellini F, Braido F, et al. Randomized controlled trials and real life studies. Approaches and methodologies: a clinical point of view. Pulm Pharmacol Ther 2014; 27: 129-138.

33 Irony TZ. The "utility" in composite outcome measures. Measuring what is important to patients. JAMA 2017; 318: $1820-1821$.

34 Parthasarathy S. The positive and negative about positive airway pressure therapy. Am J Respir Crit Care Med 2016; 194: 535-536.

35 Kohler M. Deleterious systemic effects of OSA: how much evidence do we need? Thorax 2015; 70: 817-818

36 Mehta C, Gao P, Bhatt DL, et al. Optimizing trial design. Sequential, adaptive, and enrichment strategies. Circulation 2009; 119: 597-605.

37 McMurray JJV, Packer M, Desai AS, et al. Angiotensin-neprilysin inhibition versus enalapril in heart failure. N Engl J Med 2014; 371: 993-1004.

38 Heinzer R, Vat S, Marques-Vidal P, et al. Prevalence of sleep disordered breathing in the general population: the HypnoLaus study. Lancet Respir Med 2015; 3: 310-318.

39 Drager LF, McEvoy RD, Barbe F, et al. Sleep apnea and cardiovascular disease: lessons from recent trials and need for team science. Circulation 2017; 136: 1840-1850. 